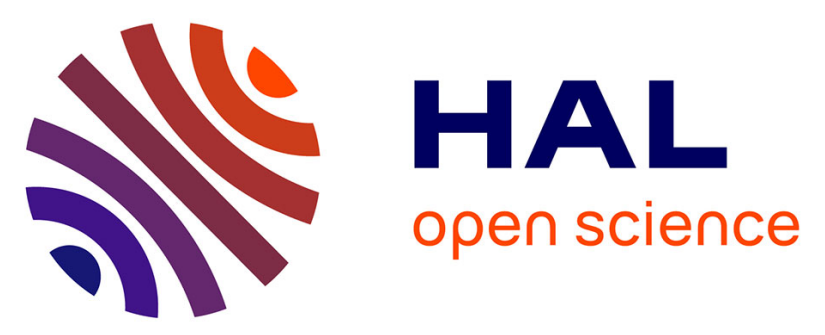

\title{
A Centralized TDMA based Scheduling Algorithm for Real-Time Communications in Vehicular Ad Hoc Networks
}

Mohamed Hadded, Paul Mühlethaler, Anis Laouiti, Leila Azouz Saidane

\section{To cite this version:}

Mohamed Hadded, Paul Mühlethaler, Anis Laouiti, Leila Azouz Saidane. A Centralized TDMA based Scheduling Algorithm for Real-Time Communications in Vehicular Ad Hoc Networks. SoftCom 2016, Sep 2016, Split Croatia. hal-01379219

\section{HAL Id: hal-01379219 https://hal.science/hal-01379219}

Submitted on 11 Oct 2016

HAL is a multi-disciplinary open access archive for the deposit and dissemination of scientific research documents, whether they are published or not. The documents may come from teaching and research institutions in France or abroad, or from public or private research centers.
L'archive ouverte pluridisciplinaire HAL, est destinée au dépôt et à la diffusion de documents scientifiques de niveau recherche, publiés ou non, émanant des établissements d'enseignement et de recherche français ou étrangers, des laboratoires publics ou privés.

$$
\text { Copyright }
$$




\title{
A Centralized TDMA based Scheduling Algorithm for Real-Time Communications in Vehicular Ad Hoc Networks
}

\author{
Mohamed Hadded*†‡, Paul Muhlethaler ${ }^{\dagger}$, Anis Laouiti* and Leila Azouz Saidane ${ }^{\ddagger}$ \\ *SAMOVAR, Télécom SudParis, CNRS, Université Paris-Saclay, 9 rue Charles Fourier 91011 EVRY, France \\ ${ }^{\dagger}$ INRIA, BP 105. 78153 Le Chesnay Cedex, Paris-Rocquencourt, France \\ ${ }^{\ddagger}$ RAMSIS Team, CRISTAL Laboratory, 2010 Campus University, Manouba, Tunisia \\ \{mohamed.hadded, anis.laouiti\}@ @elecom-sudparis.eu, paul.muhlethaler@inria.fr, leila.saidane@ensi.rnu.tn
}

\begin{abstract}
As wireless technologies inside smart cars are increasing, Vehicular Ad hoc NETworks (VANETs) are becoming a promising way to enhance driver and passenger safety by enabling each vehicle to provide a warning in real time when a critical event is predicted. These applications require reliable broadcast schemes with minimum access delay and transmission collisions, which thus increase the need for an efficient Medium Access Control (MAC) protocol. However, the design of an efficient MAC protocol in VANET networks is a challenging task due to the high speed of the nodes, the frequent changes in network topology and various QoS requirements. Motivated by this observation, in this paper we present a Centralized TDMA based MAC protocol named CTMAC for real-time communications in VANETs. In our solution, Road Side Units (RSUs) are used as central coordinators to schedule and maintain time slot assignment for the vehicles in their coverage areas. In this work, we will show how interference between vehicles in the overlapping regions can be avoided without using any complex spectrum mechanisms such as CDMA or OFDMA. The simulation results reveal that CTMAC significantly outperforms the VeMAC and ADHOC MAC protocols. in terms of transmission collisions and the overhead required to create and maintain the TDMA schedules.
\end{abstract}

Keywords-VANET, MAC Protocol, Ad hoc Network, TDMA, Safety applications, Real-Time, Road Side Unit.

\section{INTRODUCTION}

The continuing increase in road traffic accidents throughout the world has motivated the development of Intelligent Transportation Systems (ITS) and other applications to improve road safety and driving comfort. A communication network, called a Vehicular Ad-hoc NETwork (VANET), in which the vehicles are equipped with wireless devices has been developed to make these applications feasible. In a VANET, communications can be either Vehicle-to-Vehicle (V2V) or Vehicle-to-Infrastructure (V2I) [1]. Based on these two types of communications, a VANET can support a wide range of applications for safety (such as dangerous situation detection), for infotainment (such as Internet access and data exchange) and for traffic management (such as vehicle traffic optimization).

Since safety applications in VANETs have stringent QoS requirements, an efficient Medium Access Control (MAC) protocol that can provide a broadcast service with bounded access delays and minimum transmission collisions is required. Recently, MAC protocols, notably those that are based on the TDMA technique, have been used to enable multiple vehicles to use the same frequency channel without interfering with other vehicles' transmissions [1]. The TDMA principle consists in allocating the bandwidth to the vehicles by dividing the time into different frames and each frame is divided into several time slots. Each vehicle can access the channel during its dedicated time slot to send data messages, while it can only receive messages during the time slots reserved for other vehicles. However, many issues arise due to the high vehicle mobility in VANETs which can affect the performance of these protocols. Therefore, the scheduling mechanisms in TDMA-based MAC protocols should take into consideration the mobility features of VANETs. In this paper, we propose a Centralized TDMA-based MAC protocol (CTMAC) in which Road Side Units (RSUs) are used to coordinate channel access for the vehicles within their communication range.

The rest of the paper is organized as follows. In Section 2, we review related work. Section 3 describes the system models and presents the TDMA problems that may occur in a centralized VANET topology due to the high mobility of the nodes. Section 4 describes our TDMA-based MAC protocol, called CTMAC, and how it solves the interference problem between vehicles in the overlapping RSU regions without having to use complex broadband mechanisms such as FDMA or CDMA. Section 5 presents the simulation results and the performance evaluation. Finally, conclusions and future work are reported in Section 7.

\section{RELATED WORK}

MAC protocols generally fall into one of two broad categories: contention-based and contention-free. In contentionbased protocols, each node can try to access the channel when it has data to transmit using the carrier sensing mechanism [2]. The IEEE 802.11p [3], which is the emerging standard deployed to enable vehicular communication, is a Contention-based MAC protocol which uses a priority-based access scheme that employs both Enhanced Distributed Channel Access (EDCA) and Carrier Sense Multiple Access with Collision Avoidance (CSMA/CA) mechanisms [4]. Since the IEEE 802.11p standard is a Contention-based MAC, it cannot provide a reliable broadcast mechanism with bounded communication delay. This disadvantage is particularly detrimental in VANETs which are specifically designed to improve road safety. 
In Contention-free MAC protocols, only one vehicle can access the channel at any given time within a given neighborhood. Therefore, these protocols provide collision-free transmission with bounded access delay for real-time applications. In recent years, many TDMA-based MAC protocols have been proposed to guarantee real-time and reliable communications in VANETs while avoiding the access collision problem ${ }^{1}$ due to concurrent access to the same time slot. Each protocol has been proposed for a particular problem in a specific mobility scenario. For instance, the authors in [5] have proposed an AD HOC Medium Access Control (ADHOC MAC) to provide an efficient broadcast service for inter-vehicle communications and solve MAC issues such as the hidden-exposed terminal problem and QoS provision. ADHOC MAC is a contentionfree medium access protocol which implements a dynamic TDMA mechanism that is able to provide prompt access based on a distributed access technique, R-ALOHA (Reliable RALOHA [6]). Each vehicle can access the channel at least once in each frame by randomly selecting a time slot as its Basic CHannel (BCH). In [7], [8] Omar et al. developed and evaluated a contention-free multi-channel MAC protocol proposed for VANETs. This protocol supports efficient onehop and multi-hop broadcast services on the control channel without the hidden terminal problem caused by node mobility. These broadcast services are presented in [5] for ADHOC MAC. VeMAC reduces the collision rate by assigning disjoint sets of time slots to vehicles moving in opposite directions (Left, Right) and to RSUs. Since ADHOC MAC and VeMAC are fully distributed protocols, an access collision problem can occur between vehicles trying to access the same time slots.

Many alternatives exist to mitigate access collision between vehicles trying access the channel at the same time by using a central coordinator (i.e. RSU). For instance, Guo et al. in [9] propose an Adaptive Collision-Free MAC (ACFM) protocol based on a centralized dynamic time slot reservation mechanism. In ACFM, each frame is divided into a fixed number of time slots: one RSU Slot (RS) which is used by an RSU to broadcast control messages to the vehicles within its coverage area and 36 Data Slots (DS) which can be used by the vehicles to broadcast their beacon data to their neighboring vehicles. The control message that is periodically diffused by an RSU contains the DS assignment schedule for vehicles under its coverage and time synchronization information. A cycle length expansion and shrinking mechanism have been added to ACFM to ensure the fairness of the channel access protocol. When vehicle density is low in a particular subnet ${ }^{2}$, the corresponding RSU coordinator will shrink the slot assignment cycle frame by frame to avoid free slots occuring. In contrast, if vehicle density is high, the RSU will expand the assignment cycle frame by frame (at most five frames), where 36 additional free DS slots are added. However, the protocol does not handle communications between vehicles belonging to two different subnets. Moreover, due to high node mobility, the interval of time in which the vehicle stays in an RSU region is very short, which can lead to breaks in communication. In [10] and [11] Zhang et al. proposed a Unified TDMA-based Scheduling Protocol (UTSP) for V2I communications. The

\footnotetext{
${ }^{1}$ An access collision problem occurs when two or more vehicles within the same two-hop neighborhood set attempt to access the same available time slot, a problem which is likely to happen when a distributed scheme is used [1].

${ }^{2}$ The vehicles that are within the same RSU area.
}

goal of the work is to optimize the throughput for non-safety applications in VANETs. In the proposed TDMA scheduling strategy, the RSU collects the necessary information including channel state information, speed, and the Access Category (AC) characteristics of the vehicles within its communication range and then it assigns the time slots to the vehicles based on the weight function which consists of three factors, i.e. channel-quality weight factor, speed weight factor and $\mathrm{AC}$ weight factor. Since the protocol was evaluated for only one RSU, an interference problem can occur between vehicles in the overlapping regions where several RSUs are used. In this paper, we focus on this category of approach and we propose a new Centralized TDMA based MAC protocol (CTMAC) for real-time communications in VANET networks in which RSUs are used to coordinate channel access.

\section{SYSTEM MODEL AND TDMA PROBLEM STATEMENT}

\section{A. System Model}

A VANET in a highway scenario consists of a set of vehicles moving in opposite directions and under varying traffic conditions (speed, density). CTMAC is based on the assumption that each vehicle in a VANET is equipped with a GPS (Global Positioning System) or a GALLILEO receiver that also allows it to obtain an accurate real-time three-dimensional geographic position (latitude, longitude and altitude), speed and exact time. Moreover, synchronization between vehicles may be performed by using GPS timing information. Each road is divided into fixed areas of length $2 * R$, where $R$ is the transmission range. Note that each area is covered by one RSU installed at the side of the highway and in the middle of the corresponding area. In the following, we detail the slot scheduling mechanism in CTMAC and we show how this protocol can provide an efficient time slot utilization for the participating vehicles, while minimizing transmission collisions caused by the hidden node problem.

\section{B. TDMA Problem Statement}

When a centralized scheme is used to allocate a time slot, some issues should be addressed in order to implement efficient and fair centralized TDMA-based MAC protocols:

Inter-RSUs interference Each RSU adaptively creates and manages the TDMA slot reservation schedule for vehicles in its coverage. Thus, the same set of time slots can be allocated to vehicles in neighboring RSU regions. However, if there is an overlap between two neighboring RSUs that use the same frequency band, the messages broadcasted in one RSU region will affect the communications in the neighboring RSU region.

Short stay period in an $R S U$ region Due to their high speed, vehicles can join/leave an RSU region in short intervals of time, which leads to breaks in communication. Thus, the centralized MAC should ensure that a vehicle can continue to communicate at all times. Moreover, at any moment, the density of vehicles in an RSU region can vary rapidly from only a few vehicles to a high number of vehicles.

Access and merging collisions An access collision problem occurs between vehicles trying to acquire the same available time slot. On the other hand, merging collisions occur when 
two vehicles in different two-hop sets accessing the same time slot become members of the same two-hop set due to changes in their position. Generally, in VANETs, merging collisions are likely to occur in the following cases [7]:

- Vehicles moving at different speeds.

- Vehicles moving in opposite directions.

- There are RSUs installed along the road.

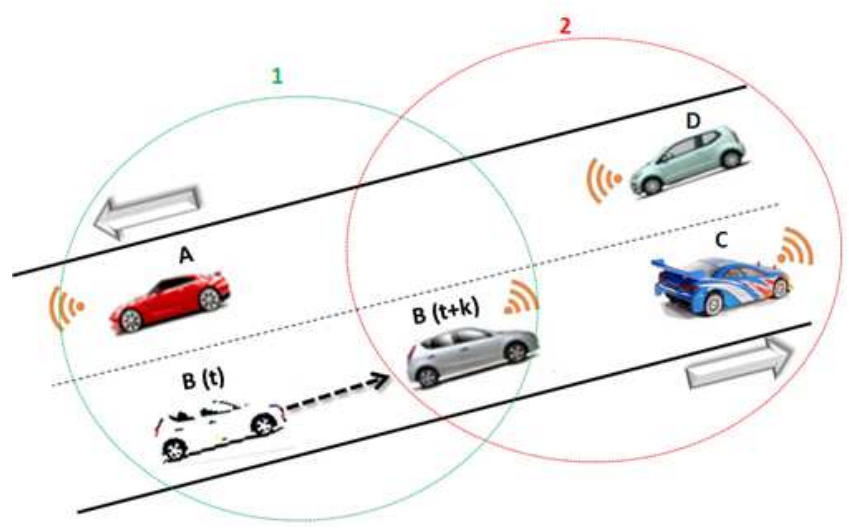

Fig. 1. Merging collision problem.

Figure 1 shows an example of the second case of the merging collision problem, when vehicle $\mathrm{B}$ in the first twohop set moving in the opposite direction to vehicle $\mathrm{D}$ in the second two-hop set is using the same time slot as B. Since $\mathrm{B}$ and $\mathrm{D}$ become members of the same two-hop set at instant $(t+k)$, a collision occurs at vehicle $\mathrm{C}$.

\section{Centralized TDMA BASEd Scheduling ALGORITHM}

\section{A. CTMAC Preliminaries}

We propose an infrastructure-based TDMA scheduling scheme which exploits the linear feature of VANET topologies. The vehicles' movements in a highway environment are linear due to the fact that their movements are constrained by the road topology. Our scheduling mechanism is also based on the assumption that the highway is equipped with some RSUs (i.e. one RSU for each $2 \times R$ meters, where $R$ is the communication range). The time slots in each TDMA frame are partitioned into two sets $S_{1}$ and $S_{2}$ associated with vehicles in two adjacent RSU areas (see Figure 2). Each frame consists of a constant number of time slots, denoted by $\tau$ and each time slot is of a fixed time duration, denoted by $s$. Each vehicle can detect the start time of each frame as well as the start time of a time slot. In the VANET studied, all the vehicles are equipped with a Global Positioning System (GPS) and thus the one-Pulse-Per-Second (1PPS) signal that a GPS receiver gets from GPS satellites can be used for slot synchronization. The first time slot either in the set $S_{1}$ or $S_{2}$ is always used by the corresponding RSU to broadcast a Slot Announcement message (SA) to the vehicles within its coverage area.

\section{B. Centralized TDMA slot scheduling mechanism}

Our centralized TDMA scheduling mechanism uses a slot reuse concept to ensure that vehicles in adjacent areas covered

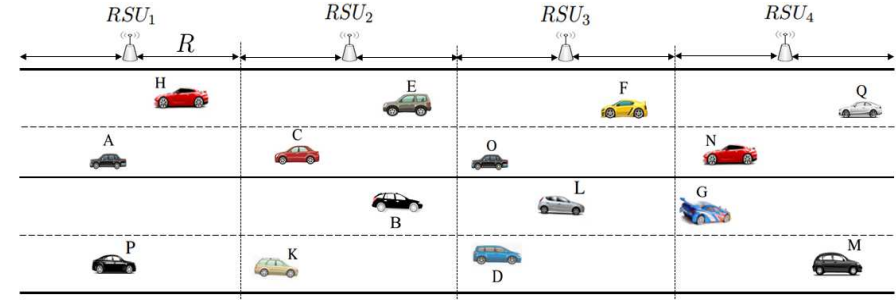

\begin{tabular}{|c|c|c|c|c|}
\hline \multicolumn{2}{|c|}{ One TDMA Frame } & & \multicolumn{2}{|c|}{ One TDMA Frame } \\
\hline$S_{1}$ & $S_{2}$ & & $S_{1}$ & $S_{2}$ \\
\hline$R S U_{1}$ & $R S U_{2}$ & $\ldots$ & $R S U_{1}$ & $R S U_{2}$ \\
\hline$R S U_{3}$ & $R S U_{4}$ & $\ldots$ & $R S U_{3}$ & $R S U_{4}$ \\
\hline : & : & & & : \\
\hline$R S U_{n-1}$ & $R S U_{n}$ & $\ldots$ & $R S U_{n-1}$ & $R S U_{n}$ \\
\hline
\end{tabular}

Fig. 2. TDMA slots scheduling mechanism of CTMAC.

by two RSUs have a collision-free schedule. The channel time is partitioned into frames and each frame is further partitioned into two sets of time slots $S_{1}$ and $S_{2}$. These sets are associated with vehicles moving in the adjacent RSU areas. These sets of time slots are reused along the highway in such a way that no vehicles belonging to the same set of two-hop neighbors ${ }^{3}$ using the same time slot. As shown in Figure 2, the vehicles in the coverage area of $R S U_{1}$ and those in the coverage area of $R \mathrm{RU}_{2}$ are accessing disjoint sets of time slots. As a result, the scheduling mechanism of CTMAC can decrease the collision rate by avoiding the inter-RSUs interference without using any complex band. Each active vehicle keeps accessing the same time slot on all subsequent frames unless it enters another area covered by another RSU or a merging collision problem occurs. Each vehicle uses only its allocated time slot to transmit its packet on the control channel.

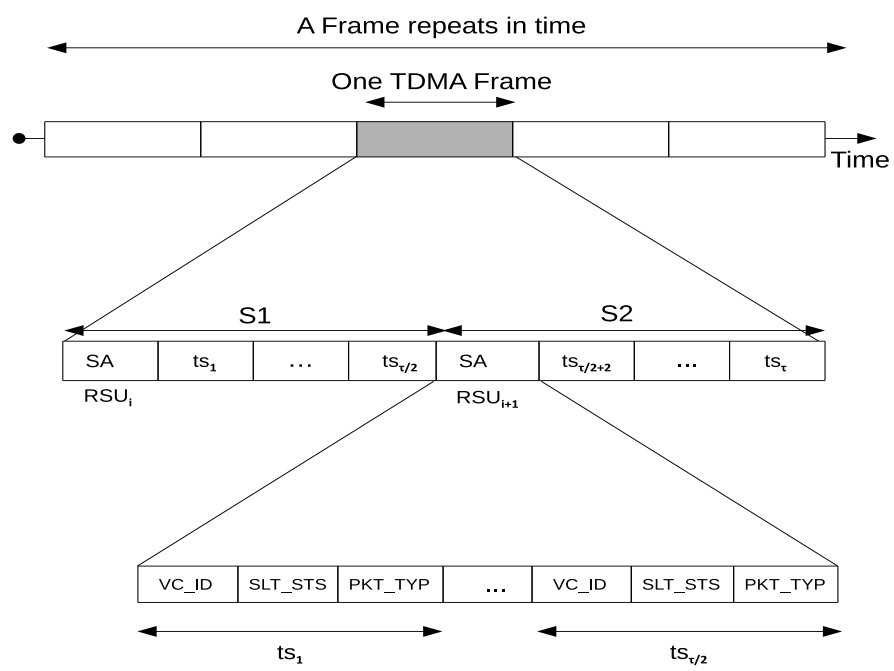

Fig. 3. Frame Information (FI) structure.

In CTMAC, each RSU constructs and maintains a Frame Information (FI) of length equal to the number of time slots

\footnotetext{
${ }^{3}$ The set of two-hop neighbors of any vehicle $x$ is the set of vehicles that can be reached at a maximum of two hops from vehicle $x$
} 
per frame, $\tau$. The FI consists of a set of ID Fields (IDFs) and each one is dedicated to the corresponding time slot of a frame. The FI structure is shown in Figure 3. Each IDF consists of three fields: VC_ID, SLT_STS and PKT_TYP. The VC_ID field contains the ID of the vehicle that is accessing this slot. The SLT_STS field contains the status of each slot which indicates whether the slot is Idle, Busy or in Collision. Finally, the PKT_TYP field indicates the type of packet transmitted by the vehicle, i.e. periodic information or event-driven safety messages. Unlike the VeMAC and ADHOC MAC protocols, in the CTMAC protocol, only the RSU nodes periodically broadcast their frame information and each vehicle will update its frame information based on the packet transmitted by its RSU. However, a vehicle broadcasts its frame information only when an access collision problem is detected.

At the end of each frame, each RSU $u$ can determine the set of free time slots based on its frame information, denoted by $F(u)$. When an RSU has one or more available time slots, it announces that by broadcasting a Slot Announcement (SA) message containing its identity $\left(S A->N O D E \_I D\right)$ to all the vehicles in its coverage area. When a vehicle receives an SA message, and if it wishes to access the channel, it tries to get the attention of the RSU by sending it a Slot REQuest message (SREQ) including its identity. Algorithm 1 outlines the details of the slot reservation mechanism. $v$ represents the vehicle that needs to reserve a time slot, timer $_{1}$ is a timer and $T S(v)$ is the time slot that is successfully acquired by vehicle $v$. When an RSU receives the SERQ message, it checks whether there is an available time slot and, if there is, the RSU sends a Slot REPly message (SREP) to the corresponding vehicle including the slot index $\left(S R E P->S L T_{-} I D\right)$. After the reception of the SREP, the vehicle $v$ starts to broadcast its message during its time slot, $T S(v)=S R E P->S L T_{-} I D$. Otherwise, if the timer expires and no response has been received from the RSU (lines 12-14), the vehicle $v$ will repeat the same steps.

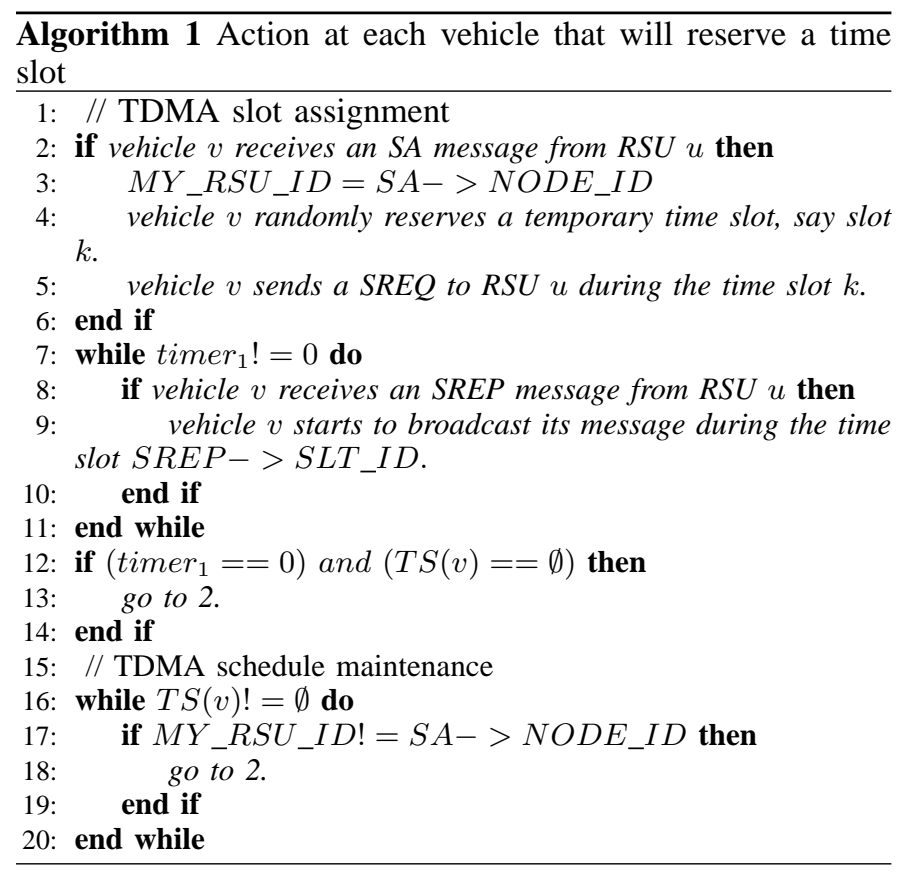

If a vehicle receives an SA message from another RSU (line
17), the vehicle will send an SREQ to allocate a new time slot and if it receives an SREP from the RSU it will release its current time slot and it will start to broadcast its packet during the time slot allocated by the new RSU. Moreover, when an RSU does not receive a message from a vehicle $v$ during its slot, it considers that it has left its coverage area and it releases its time slot. Algorithm 2 outlines the behavior of our scheme during the procedure of slot scheduling at the RSU.

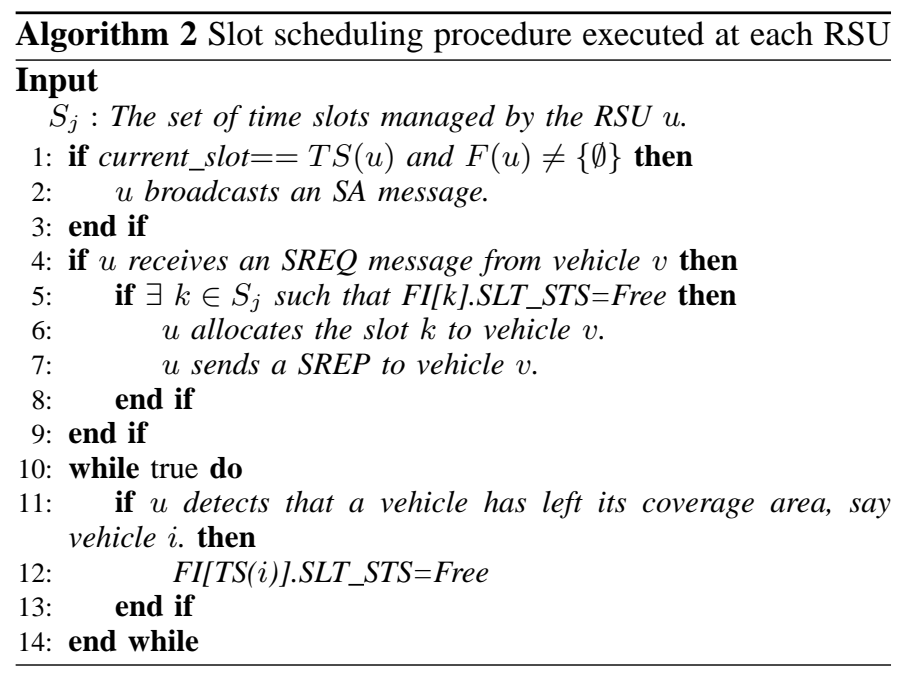

\section{Access Collision Avoidance}

In Figure 4, we show an example of Access Collision Avoidance (ACA) mechanism implemented by CTMAC. The VANET scenario consists of 4 vehicles identified from ( $v 1$ to v4) and one RSU, using a CTMAC's scheduling represented by vectors (one vector for each node) of length equal to 5. Each element of a vector represents one time slot that can be used by only one node to send messages. We assume that two vehicles $v_{3}$ and $v_{4}$ have sent respectively their SREQ1, SREQ2 to RSU1 during the same time slot $(t s=3)$ in frame $i$, as shown in Figure 4. The RSU did not confirm their reservations because their packets collided. Since the neighboring vehicles $v_{1}$ and $v_{2}$ have respectively received SREQ1 and SREQ2 without a collision problem, they will update their frame information by adding the vehicles $v_{3}$ and $v_{4}$ and then will send their new captured frame information to the RSU1 during the time slot $t s=2$ and $t s=3$ in frame $i+1$, respectively. Upon reception, the RSU1 observes that $v_{3}$ and $v_{4}$ are trying access the channel and to prevent the access collision problem occurring again, it will broadcast frame information including new time slots for vehicles $v_{3}$ and $v_{4}$ during the time slot $t s=1$ in frame $i+2$. When all vehicles receive a packet transmitted by an RSU1, they will update their FIs.

\section{SIMULATION RESULTS AND PERFORMANCE EVALUATION}

\section{A. Simulation Scenarios}

We generated a realistic VANET environment by selecting a real highway area from a digital map which took into account lane directions. Figure 5 shows a metropolitan area from a map of San Jose (California) of size $3000 \mathrm{~m} \times 100 \mathrm{~m}$ exported from OpenStreetMap (OSM) and edited using Java 


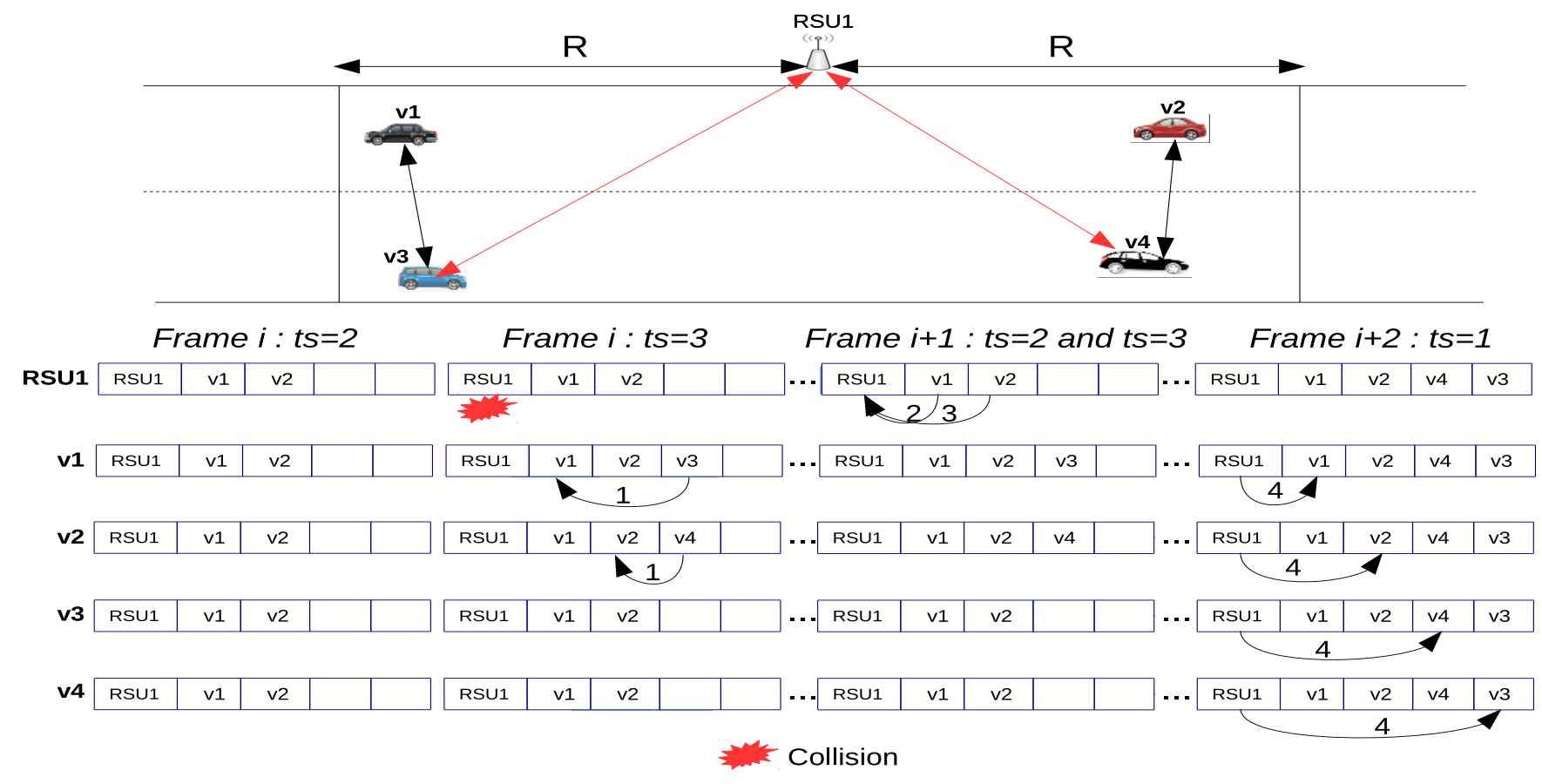

Fig. 4. Access Collision Avoidance.

OpenStreetMap Editor (JOSM). Then MOVE and SUMO [12] were used respectively to generate vehicular traffic scenarios and to simulate the area with vehicular traffic. To do that, we defined a vehicle flow which described a swarm of vehicles in each direction. The parameters of each vehicle flow consisted of the maximum number of vehicles, the starting road and destination of the flow, the time to start and end the flow. We assigned a random speed to each vehicle between $120 \mathrm{~km} / \mathrm{h}$ and $150 \mathrm{~km} / \mathrm{h}$. Then the traffic traces generated by SUMO were used in the $n s 2.34$ simulator. The simulation parameters used in our experiments are summarized in Table I.

We have used a parameter, called RSU Coverage Occupancy (RCO) [8], equal to $N_{v} \times \frac{2 R}{L_{h}} \times \frac{2}{\tau}$ in a highway scenario, where $N_{v}$ is the total number of active vehicles, $R$ is the communication range and $L_{h}$ is the length of the highway. CTMAC is evaluated based on the following metrics:

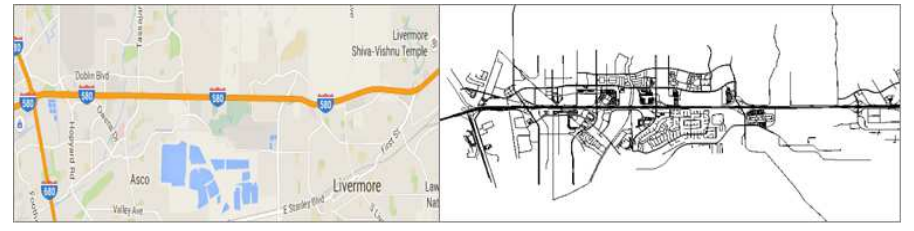

Fig. 5. San Jose (California) urban area captured from Google Maps (left) and exported to a VANET network topology by using MOVE/SUMO (right)

1) The access collision rate: is defined as the average number of access collisions per slot per RSU coverage area.

2) The merging collision rate: is defined as the average number of merging collisions per slot per RSU coverage area.

3) The packet loss rate: is defined as the average of
TABLE I. Simulation parameters

\begin{tabular}{cc}
\hline Parameter & Value \\
\hline Highway length & $2.5 \mathrm{~km}$ \\
Lanes/direction & 2 \\
Vehicle speed & $120 \mathrm{~km} / \mathrm{h}$ \\
Speed standard deviation $(\sigma)$ & $30 \mathrm{~km} / \mathrm{h}$ \\
Transmission range $(R)$ & $310 \mathrm{~m}$ \\
Slots/frame $(\tau)$ & 100 \\
Slot duration $(\mathrm{s})$ & $0.001 \mathrm{~s}$ \\
Simulation time & $120 \mathrm{~s}$ \\
\hline
\end{tabular}

the total number of vehicles that do not successfully receive messages to the total number of vehicles within communication range of the transmitter.

4) Overhead: which is the rate of control packets used to allocate a time slot as well as to maintain the TDMA schedules.

\section{B. CTMAC performance evaluations}

Figure 6 shows the rate of merging collisions for the CTMAC, VeMAC and ADHOC MAC protocols when varying the RSU Coverage Occupancy (RCO). We can note from this figure that CTMAC prevents more merging collisions than ADHOC MAC and VeMAC even for a high RCO. These results can be explained by the fact that CTMAC separates neighboring RSU areas by assigning disjoint sets of time slots to vehicles traveling in these areas. However, in VeMAC, the vehicles that cannot access a time slot from the set of slots reserved for its direction, will attempt to access any available time slot reserved for vehicles moving in the opposite direction. As a result, the merging-collisions occur frequently in VeMAC when traffic density is high, especially when the number of vehicles in each direction is not equal. However, these results might well be expected for the ADHOC MAC 
protocol since all vehicles randomly acquire a time slot in the frame without considering which direction they are moving in, which could make it susceptible to the merging collisions problem in highway scenarios where the vehicles are moving in opposite directions.

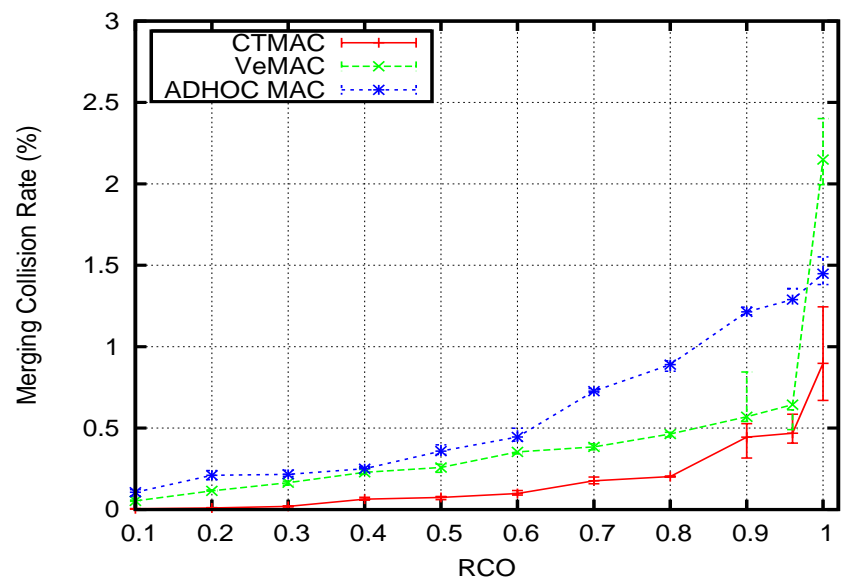

Fig. 6. The rate of merging collisions.

Figure 7 shows the access collision rates of the three TDMA based MAC protocols under consideration. For a $R C O \leq 0.6$, all the protocols have almost the same access collisions rate, while for a $R C O \geq 0.7$, CTMAC starts to perform better than VeMAC and ADHOC MAC. These results can be explained by the fact that VeMAC and ADHOC MAC have achieved a higher rate of merging collision compared to CTMAC. Indeed, upon detecting of merging-collisions, the nodes in collision should release their time slots and request new ones, which can reproduce access-collisions. Moreover, as discussed in Section 4, by using the RSU as a central coordinator to schedule and maintain time slot assignment for the vehicles in its coverage area one can prevent the access collision problem occurring more than once between the same vehicles that are trying to access the channel.

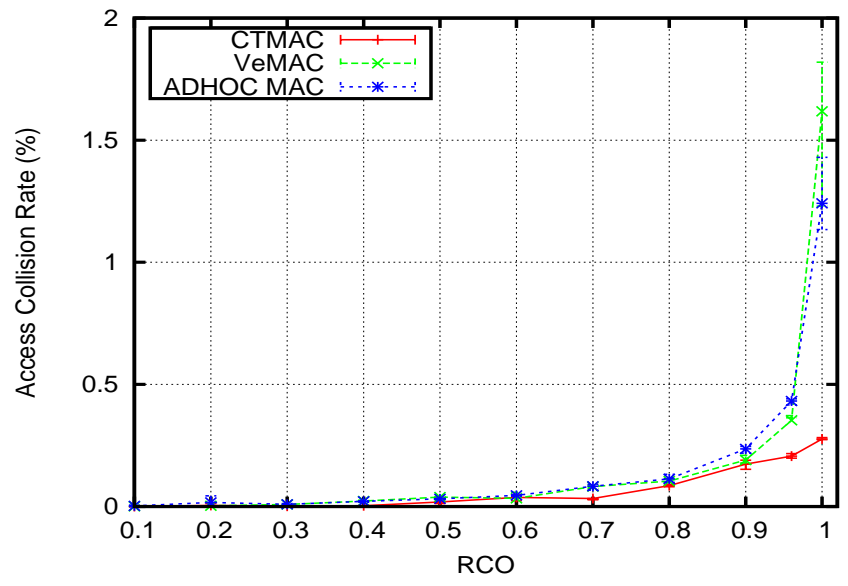

Fig. 7. The rate of access collisions.

The packet loss rates of the three MAC protocols under consideration are shown in Figure 8 . It can be seen that our
MAC protocol has the lowest packet loss rate, especially for a high RCO, due to its ability to handle the access and merging collision problem. For instance, at a $R C O=1$, the VeMAC and ADHOC MAC protocols show approximately $103.4 \%$ and $90.1 \%$ higher rates of packet loss than the CTMAC protocols, respectively.

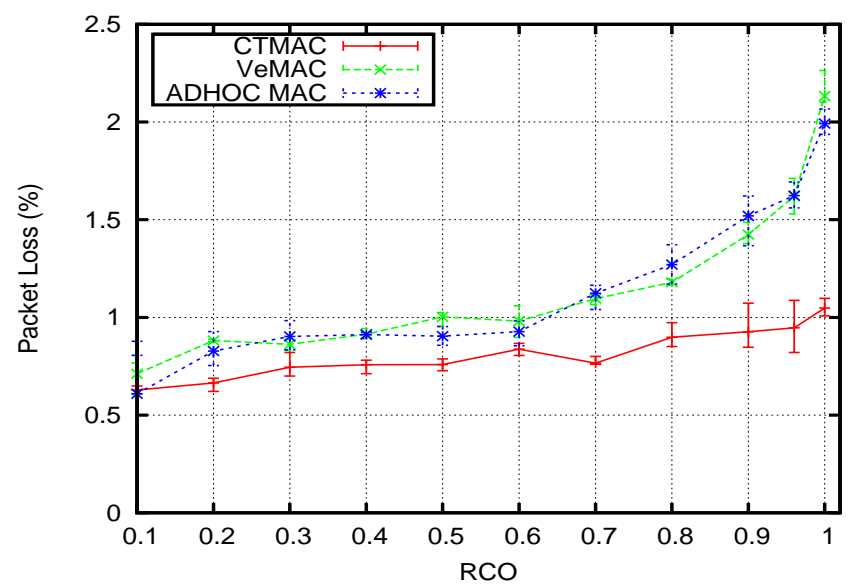

Fig. 8. The rate of packet loss under various traffic densities.

Figure 9 shows the overhead (Mega octet) generated by each protocol during $120 \mathrm{~s}$. We can see from this figure that CTMAC has reduced the overhead compared to VeMAC and ADHOC MAC. For instance, at $\mathrm{RCO}=0.96$, the overhead is reduced by respectively $85.52 \%$ and $83.81 \%$ on average when CTMAC is used compared to the VeMAC and ADHOC MAC protocols. These results can be explained by the fact that CTMAC uses the RSUs to assign time slots and to disseminate the FI and then all vehicles within their communication range will update their slot schedule tables based on the FI received, in contrast to VeMAC and ADHOC MAC that are fully distributed protocols in which each vehicle periodically broadcasts the FI to its direct neighbors in order to maintain the TDMA schedule table.

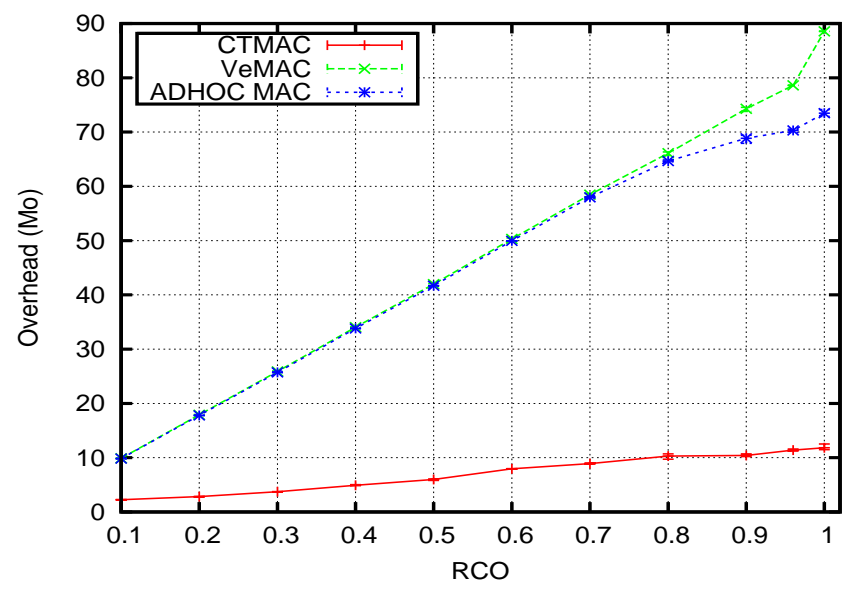

Fig. 9. TDMA scheduling Overhead. 


\section{CONCLUSION}

In this paper, we proposed a centralized TDMA-based MAC protocol, named CTMAC in which an RSU is used as a local channel coordinator for the vehicles within its communication range. The ways that slots are allocated and reused between the RSU's coverage areas are designed to avoid collisions caused by the interference problem between vehicles in the overlapping regions. The simulation results show that, compared to VeMAC and ADHOC MAC protocols, CTMAC has succeeded to provide a smaller rate of access and merging collisions as well as the overhead required to create and maintain the TDMA schedules.

In future work, CTMAC will be extended to support multichannel operation and to provide a reliable multi-hop broadcast service on the control channel. Moreover, we will carry out extensive simulations to compare it with the IEEE 802.11p standard. In addition, the future version of CTMAC will allow each vehicle to acquire more than one time slot per frame on the transmission channel.

\section{REFERENCES}

[1] M. Hadded, P. Muhlethaler, A. Laouiti, R. Zagrouba, and L. A. Saidane, "Tdma-based mac protocols for vehicular ad hoc networks a survey, qualitative analysis and open research issues," IEEE Communications Surveys Tutorials, vol. 17, no. 4, pp. 2461-2492, Jun. 2015.

[2] F. Ye, R. Yim, J. Zhang, and S. Roy, "Congestion control to achieve optimal broadcast efciency in vanets," in IEEE International Conference on Communications (ICC), Cape Town, South Africa, May 2010, pp. $1-5$.

[3] $802.11 p$-2010, IEEE standard for information technology - Telecommunications and information exchange between systems - local and metropolitan area networks - specific requirements part 11 : Wireless LAN medium access control (MAC) and physical layer (PHY) and physical layer (PHY) specifications amendment 6 : Wireless access in vehicular environments Std., 2010

[4] R. Uzcátegui and G. Acosta-Marum, "Wave: A tutorial," IEEE Communications Magazine, vol. 47, no. 5, pp. 126-133, May 2009.

[5] F. Borgonovo, A. Capone, M. Cesana, and L. Fratta, "Adhoc mac: new mac architecture for ad hoc networks providing efficient and reliable point-to-point and broadcast services," Wireless Networks, vol. 10, no. 4, pp. 359-366, 2004.

[6] _ "Rr-aloha, a reliable r-aloha broadcast channel for ad-hoc intervehicle communication networks," in IEEE IFIP Annual Mediterranean Ad Hoc Networking Workshop (Med-Hoc-Net), Baia Chia, Italy, 2002.

[7] W. Zhuang, H. A. Omar, and L. Lio, "Vemac: A novel multichannel mac protocol for vehicular ad hoc networks," in IEEE Conference on Computer Communications Workshops (INFOCOM WKSHPS), Shanghai, China, Aug. 2011, p. 413418.

[8] H. A. Omar, W. Zhuang, and L. Li, "Evaluation of vemac for v2v and v2r communications under unbalanced vehicle traffic" in IEEE Vehicular Technology Conference (VTC Fall), Qubec City, Canada, Sep. 2012, pp. 1-5.

[9] W. Guo, L. Huang, L. Chen, H. Xu, and J. Xi, "An adaptive collisionfree mac protocol based on tdma for inter-vehicular communication," in International Conference on Wireless Communications and Signal Processing (WCSP), Anhui, China, Oct. 2012, pp. 1-6.

[10] R. Zhang, J. Lee, X. Shen, X. Cheng, L. Yang, and B. Jiao, "A unified tdma-based scheduling protocol for vehicle-to-infrastructure communications," in International Conference Wireless Communications and Signal Processing (WCSP), Hangzhou, Oct. 2013, pp. 1-6.

[11] R. Zhang, X. Cheng, L. Yang, X. Shen, and B. Jiao, "A novel centralized tdma-based scheduling protocol for vehicular networks," IEEE Transactions on Intelligent Transportation Systems, vol. PP, no. 99, pp. 1-6, Aug. 2014.
[12] F. Karnadi, Z. Mo, and K. chan Lan, "Rapid generation of realistic mobility models for vanet," in IEEE WCNC, Hong Kong, China, Mar. 2007, pp. 2506-2511. 